\title{
Interaction of turbulence with the leading-edge stagnation point of a thin aerofoil
}

\author{
L ORNA J. A Y T ON †and N. PEAKE \\ Department of Applied Mathematics and Theoretical Physics, University of Cambridge, \\ Wilberforce Road, CB3 0WA, UK
}

(Received 6 May 2016)

An asymptotic model is constructed to analyse the interaction of turbulence generated far upstream with a thin elliptic-nosed solid body in uniform flow. The leading-edge stagnation point causes significant deformation of incident vorticity, and hence our analysis focuses on the region of size scaling with the nose radius close to the stagnation point. Rapid distortion theory is used to separate the flow field generated by a single unsteady gust perturbation into a convective non-acoustic part, containing the evolution of the upstream vortical disturbance, and an acoustic part generated by the interaction of the vorticity with the solid surface, as is typical in gust-aerofoil interaction theory. Using single-frequency gust response solutions, along with a von Karman energy spectrum, we find the turbulent pressure spectrum generated by homogeneous isotropic turbulence incident from far upstream. Both high- and low-frequency gusts are considered to allow approximations to be found for the turbulent pressure spectra close to the leading edge, and far from the body close to the incident stagnation streamline. Good agreement is shown between the asymptotic results for the near- and far-field leading-edge turbulent pressure spectra and recent experimental findings.

\section{Introduction}

The problem of unsteady vorticity-aerofoil interaction in steady uniform flow has been considered analytically (Myers \& Kerschen 1997; Tsai 1992; Goldstein \& Atassi 1976), computationally (Hixon et al. 2006; Allampalli et al. 2009) and experimentally (Geyer et al. 2012; Mish \& Devenport 2006), since understanding this process is key to predicting the noise generated by blade rows within aeroengines. Computational methods can obtain results for realistic situations at moderate frequency, but are difficult for high-frequency incident perturbations, and hence analytic results are sought for high-frequency gustaerofoil interactions. Analytic methods, however, require significant assumptions before progress can be made; typically the aerofoil is assumed to be either a flat plate or to have small (but non-zero) thickness, camber and angle of attack, all scaling with a small asymptotic shape parameter, $\tau \ll 1$. The scaled frequency of an incident gust, $k$, is assumed to either be large, $k \gg 1$, such that $\tau k=O(1)$ as done by Myers \& Kerschen (1997), or is assumed to be small, $k \ll 1$ (Amiet 1974).

In practice both the far-field sound and the unsteady surface pressure are of interest. For aerofoils with $O(\tau)$ camber angle and thickness, the stagnation point of the steady flow is typically an $O\left(\tau^{2}\right)$ distance from the aerofoil nose (Van Dyke 1975) which is negligible during asymptotic analysis. Within the limits of standard thin-aerofoil theory (analytic and numeric), there is a singularity in the analytic surface perturbation pressure at the stagnation point, which violates the assumption that the unsteady perturbation 
pressure is small, however it can be shown that the unsteady pressure calculated along the rest of the aerofoil surface and in the far-field agree with experimental findings. This leading-edge singularity is an anomaly that requires closer attention in order to understand the turbulent interactions close to the stagnation point.

In this paper we attempt to correct the leading-edge singularity and investigate the true behaviour of turbulence at the stagnation point by considering the evolution and interaction of an arbitrary gust in a steady uniform flow past a thin elliptic cylinder (extending infinitely in the spanwise direction), specifically concentrating on the region close to the leading edge of the body, and in the far field at small angles away from the incident stagnation-point streamline (which shall also be referred to as the zerostreamline). The thickness of the ellipse is parametrised by $\tau \ll 1$, and the frequency of the gust is parameterised by $k$ which we permit to either be large, $k \gg 1$, or small, $k \ll 1$. The leading-order contribution to the acoustic solution can be dependent on a multiplicative combination of thickness and frequency, which ensures that the thickness parameter can remain present in the analysis even to leading order, which is not the case in previous analytic solutions (Myers \& Kerschen 1997; Tsai 1992). We use the solution for single-frequency gust interactions to investigate the effects of the leading-edge stagnation point on weak homogeneous isotropic turbulence in uniform flow, and obtain an analytic prediction for the inviscid far-field and surface pressure spectra close to the stagnation streamline.

Hunt (1973) calculates the surface pressure spectrum of a circular cylinder. Later Durbin \& Hunt (1980) generalise Hunt's result for a bluff two-dimensional body, e.g. an elliptic cylinder; their work however does not allow for an asymptotic scaling in thickness, and instead the only asymptotic parameter used in Durbin \& Hunt (1980) relates to the integral lengthscale of the turbulence far upstream and the incident frequency. By assuming the ellipse has $O(1)$ thickness (or more precisely has an $O(1)$ aspect ratio) and calculating just the leading-order pressure spectrum, Durbin \& Hunt (1980) have ignored the possibility of an asymptotic reordering of terms due to small ellipse thickness. Likewise, Durbin \& Hunt (1980) do not restrict attention to a small region close to the stagnation point and instead assume $O(1)$ values of polar angle as measured from the upstream direction (except for calculations at the stagnation point itself). It is these new allowable scalings that we permit in this paper, and present a leading-order approximation for the turbulent pressure spectrum that accounts for high or low turbulent frequency, large or small integral lengthscale of turbulence, and a thin body as is applicable for aeroacoustics. We specifically focus on a small region close to the stagnation point. We therefore anticipate finding a different leading-order result for the pressure spectrum than that found in Durbin \& Hunt (1980).

In Section 2 we discuss the interaction of a single-frequency gust with the nose of a thin ellipse, obtaining the acoustic response in both high- and low-frequency cases. In Section 3 we use the single-frequency solutions to determine the one-dimensional turbulent pressure spectra close to the leading-edge stagnation point, and in the far field near the zero streamline. Section 3.1 discusses the far-field spectra at high reduced frequency ( $k l$, where $l$ is the integral lengthscale of the turbulence), whilst Section 3.2 considers high and low reduced frequency surface spectra. Section 4 contains results and comparison with experimental data, and we discuss conclusions in Section 5.

\section{Acoustic Response for a Single Frequency Gust}

We begin by considering the interaction of an unsteady gust with a thin ellipse of minor axis $R^{*}\left(1-b^{2}\right)$ and major axis $R^{*}\left(1+b^{2}\right)$ in steady low Mach number uniform 


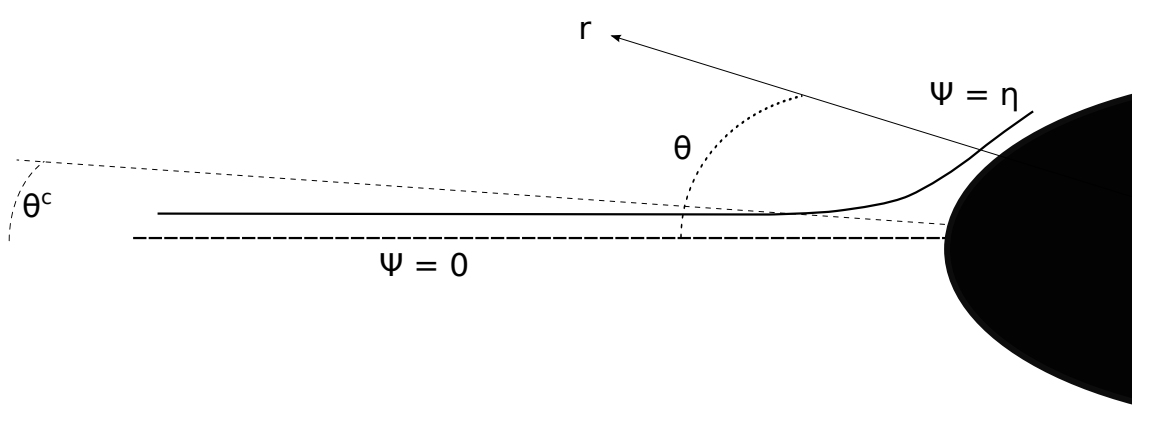

Figure 1: Diagram of the coordinate system at the leading edge.

flow, $\boldsymbol{U}=U_{\infty}^{*} \boldsymbol{e}_{x}$ far upstream, at zero angle of attack. We non-dimensionalise velocities by $U_{\infty}^{*}$ and lengths by $R^{*}$, and define thickness parameter, $\tau=\left(1-b^{2}\right)\left(1+b^{2}\right)^{-1}$, which we take to be small. In (cylindrical) polar coordinates, $r$ measures the radial distance from the centre of the ellipse and $\theta$ measures the angle clockwise from the upstream direction, as illustrated in Figure 1. We label the stagnation streamline of the steady flow $\Psi=0$, and a streamline formally bounding our analysis away from the stagnation streamline as $\Psi=\eta$, where $\eta \ll 1$. The constant $\theta^{c}$ provides a lower limit of $\theta$ values, formally bounding our analysis away from $\theta=0$, and denotes the angle at which the $\eta$ streamline first deviates from the horizontal.

\subsection{Governing Equations for the Acoustic Response}

We write an unsteady harmonic disturbance upstream as a Fourier integral

$$
\boldsymbol{u}_{\infty}\left(\boldsymbol{x}-\hat{\boldsymbol{e}}_{x} t\right)=\int \boldsymbol{A}(\boldsymbol{k}) \mathrm{e}^{\mathrm{i} \boldsymbol{k} \cdot \boldsymbol{x}-k_{1} t} d \boldsymbol{k}
$$

where $\boldsymbol{u}_{\infty}$ is solenoidal so $\boldsymbol{A} \cdot \boldsymbol{k}=0 \quad \forall \boldsymbol{k}$. We write $\boldsymbol{k}=k \boldsymbol{k}^{\dagger}=\left(k_{1}, k_{2}, k_{3}\right)$, where $k$ is our asymptotic frequency parameter. We initially consider just one Fourier mode given by

$$
\boldsymbol{u}_{\infty}\left(\boldsymbol{x}-\hat{\boldsymbol{e}}_{x} t\right)=\boldsymbol{A}(\boldsymbol{k}) \mathrm{e}^{\mathrm{i}\left[k_{1}(x-t)+k_{2} y+k_{3} z\right]}
$$

far upstream. It follows from Goldstein (1978) that the vortical components of the velocity are

$$
\begin{aligned}
& u_{r}^{(I)}=\left(A_{1} \frac{\Delta}{r}+A_{2} \frac{\Psi}{r}\right) \mathrm{e}^{\mathrm{i}\left[k_{1}(\Delta-t)+k_{2} \Psi+k_{3} z\right]}, \\
& u_{\theta}^{(I)}=\left(A_{1} \frac{1}{r} \frac{\partial \Delta}{\partial \theta}+A_{2} \frac{1}{r} \frac{\partial \Psi}{\partial \theta}\right) \mathrm{e}^{\mathrm{i}\left[k_{1}(\Delta-t)+k_{2} \Psi+k_{3} z\right]}, \\
& u_{z}^{(I)}=A_{3} \mathrm{e}^{\mathrm{i}\left[k_{1}(\Delta-t)+k_{2} \Psi+k_{3} z\right]},
\end{aligned}
$$

where $\Delta$ is the drift function of the steady flow (non-dimensionalised by $U^{*} / R^{*}$ ), which was first discussed by Darwin (1953), then later defined by Lighthill (1956) as

$$
\Delta=x+\int_{-\infty}^{x}\left(\frac{1}{U_{x}}-1\right) d x .
$$


This function is the difference between the time taken for a fluid particle to travel from far upstream to position $x$ along a streamline in the given flow, compared to the time taken to reach the same position in uniform flow.

When the total unsteady velocity field, $\boldsymbol{u}$, is written as

$$
\boldsymbol{u}=\boldsymbol{\nabla} \phi+\boldsymbol{u}^{(I)},
$$

$\phi$ contains the acoustic response to the incident gust and satisfies the acoustic equation arising from rapid distortion theory,

$$
\frac{D_{0}}{D t}\left(\frac{1}{c^{2}} \frac{D_{0}}{D t}\right) \phi-\frac{1}{\rho} \boldsymbol{\nabla} \cdot(\rho \boldsymbol{\nabla} \phi)=\frac{1}{\rho} \boldsymbol{\nabla} \cdot\left(\rho \boldsymbol{u}^{(I)}\right),
$$

subject to the condition of zero normal velocity,

$$
\boldsymbol{\nabla} \phi \cdot \boldsymbol{n}=-\boldsymbol{u}^{(I)} \cdot \boldsymbol{n},
$$

on the solid surface. We have defined $\frac{D_{0}}{D t}$ by $\frac{\partial}{\partial t}+\boldsymbol{U} \cdot \boldsymbol{\nabla}$. The steady density, $\rho=1+$ $M^{2} \rho_{1}+O\left(M^{4}\right)$, and $c$, the speed of sound, given by

$$
c^{2}=\frac{1}{M^{2}}\left[1-\frac{\gamma-1}{2} M^{2}\left(|\boldsymbol{U}|^{2}-1\right)\right] \equiv M^{-2}\left(1+M^{2} c_{1}^{2}\right)+O\left(M^{2}\right)
$$

are expanded as an asymptotic series in the uniform flow Mach number at infinity, $M$, since we restrict attention to low Mach number flows only. The constant $\gamma$ is the ratio of specific heats.

Using Eq. (2.3) we can write the source term in $(2.6 a)$ as

$$
\frac{1}{\rho} \boldsymbol{\nabla} \cdot\left(\rho \boldsymbol{u}^{(I)}\right)=\left(H_{0}+\mathrm{i} H_{1}\right) \mathrm{e}^{\mathrm{i}\left[k_{1}(\Delta-t)+k_{2} \Psi+k_{3} z\right]},
$$

where $H_{0,1}$ are defined by

$$
\begin{aligned}
H_{0}= & \frac{A_{1}}{r} \frac{\partial \Delta}{\partial r}+\frac{A_{2}}{r} \frac{\partial \Psi}{\partial r}+\frac{A_{1}}{r^{2}} \frac{\partial^{2} \Delta}{\partial \theta^{2}}+\frac{A_{2}}{r^{2}} \frac{\partial^{2} \Psi}{\partial \theta^{2}} \\
& +M^{2}\left[\left(\frac{A_{1}}{r} \Delta+\frac{A_{2}}{r} \Psi\right) \frac{\partial \rho_{1}}{\partial r}+\frac{1}{r} \frac{\partial \rho_{1}}{\partial \theta}\left(\frac{A_{1}}{r} \frac{\partial \Delta}{\partial \theta}+\frac{A_{2}}{r} \frac{\partial \Psi}{\partial \theta}\right)\right], \\
H_{1}= & A_{1} k_{1}\left[\left(\frac{1}{r} \frac{\partial \Delta}{\partial \theta}\right)^{2}+\frac{\partial \Delta}{\partial r} \frac{\Delta}{r}\right]+A_{2} k_{2}\left[\left(\frac{1}{r} \frac{\partial \Psi}{\partial \theta}\right)^{2}+\frac{\partial \Psi}{\partial r} \frac{\Psi}{r}\right]+A_{3} k_{3} \\
& +A_{1} k_{2}\left[\frac{1}{r^{2}} \frac{\partial \Delta}{\partial \theta} \frac{\partial \Psi}{\partial \theta}+\frac{\Delta}{r} \frac{\partial \Psi}{\partial r}\right]+A_{2} k_{1}\left[\frac{1}{r^{2}} \frac{\partial \Delta}{\partial \theta} \frac{\partial \Psi}{\partial \theta}+\frac{\Psi}{r} \frac{\partial \Psi}{\partial r}\right] .
\end{aligned}
$$

We wish to solve $(2.6 a)$ to leading order in both high- and low-frequency regimes.

We restrict the bounding streamline, $\Psi=\eta$, Mach number, $M$, and region of interest near the leading edge (i.e. range of $\theta$ ) as follows; $\eta=O\left(\tau^{4}\right), M=O(\tau)$, and $\theta=O(\tau)$, where $\tau$ is the asymptotic thickness parameter. This restriction of $\theta$ creates an asymptotic region around the leading edge which differs from regions used in previous asymptotic gust-aerofoil analysis such as Tsai (1992) and Myers \& Kerschen (1997); in these problems the leading-edge inner regions scale on blade thickness, e.g. $r-\left(1+b^{2}\right)=O(\tau)$, but $\theta$ was assumed to be $O(1)$. For our problem, since $r$ measures the radial distance from the centre of the ellipse, we suppose $r=O(1)$.

In both high- and low-frequency cases, we set

$$
\phi=\mathrm{e}^{-\mathrm{i} k_{1} t+\mathrm{i} k_{3} z} \tilde{\phi},
$$


Interaction of turbulence with the leading-edge stagnation point of a thin aerofoil 5 and expand $\tilde{\phi}=\tilde{\phi}_{0}+M^{2} \tilde{\phi}_{1}$. Retaining terms in (2.6a) up to $O\left(M^{2}\right)$, we obtain

$$
\begin{aligned}
& M^{2}\left(-k_{1}^{2} \tilde{\phi}_{0}+2 \mathrm{i} k_{1} \boldsymbol{U}_{0} \cdot \boldsymbol{\nabla} \tilde{\phi}_{0}+\left(\boldsymbol{U}_{0} \cdot \boldsymbol{\nabla}\right)\left(\boldsymbol{U}_{0} \cdot \boldsymbol{\nabla} \tilde{\phi}_{0}\right)\right) \\
& -\boldsymbol{\nabla}^{2} \tilde{\phi}_{0}-M^{2}\left(\boldsymbol{\nabla}^{2} \tilde{\phi}_{1}+\boldsymbol{\nabla} \cdot\left(\rho_{1} \boldsymbol{\nabla} \tilde{\phi}_{0}\right)-\rho_{1} \boldsymbol{\nabla}^{2} \tilde{\phi}_{0}\right)=\left(H_{0}+\mathrm{i} H_{1}\right) \mathrm{e}^{\mathrm{i} k_{1} \Delta+\mathrm{i} k_{2} \Psi},
\end{aligned}
$$

where we have assumed the steady velocity can be written as $\boldsymbol{U}=\boldsymbol{U}_{0}+O\left(M^{2}\right)$, with $\boldsymbol{U}_{0}$ the incompressible steady flow around the ellipse, whose components are $O(1)$ under the given $\theta=O(\tau)$ scaling.

For high frequencies, $k \gg 1$, assuming $k>\tau^{-1}$ and retaining only the leading-order terms yields

$$
-M^{2} k_{1}^{2} \tilde{\phi}_{0}-\frac{1}{r^{2}} \frac{\partial^{2} \tilde{\phi}_{0}}{\partial \theta^{2}}+k_{3}^{2} \tilde{\phi}_{0}=\left(H_{0}+\mathrm{i} H_{1}\right) \mathrm{e}^{\mathrm{i} k_{1} \Delta+\mathrm{i} k_{2} \Psi}
$$

where the forcing terms, $H_{0,1}$ are taken to leading order only.

For low frequencies $k \ll 1$, the convective terms are negligible compared to the Laplacian, therefore we obtain

$$
-\frac{1}{r^{2}} \frac{\partial^{2} \tilde{\phi}_{0}}{\partial \theta^{2}}=\left(H_{0}+\mathrm{i} H_{1}\right) \mathrm{e}^{\mathrm{i} k_{1} \Delta+\mathrm{i} k_{2} \Psi},
$$

where once again the forcing terms, $H_{0,1}$ are taken to leading order only.

We see from equations (2.12) and (2.13), that to obtain a leading-order approximation for $\tilde{\phi}_{0}$, we need only calculate $H_{0,1}$ to leading order, which will be given by the incompressible steady flow solutions for $\Delta$ and $\Psi$. In particular we require these functions approximated in the small $\theta$ limit. We obtain them by conformal mapping incompressible steady uniform flow around a circular cylinder of radius 1 to our required elliptic cylinder using the Joukowski transformation. Relevant calculations can be found in Appendix A.

We now proceed to solve (2.12) and (2.13) in our high- and low-frequency regimes respectively.

\subsection{High-Frequency Solution}

We rewrite $(2.12)$ as

$$
\frac{\partial^{2} \tilde{\phi}}{\partial \theta^{2}}+k^{2} w^{2} r^{2} \tilde{\phi}=-r^{2} f(r, \theta)
$$

where $w^{2}=k_{1}^{\dagger 2} M^{2}-k_{3}^{\dagger 2}$, and

$$
f(r, \theta)=\left(H_{0}(r, \theta)+\mathrm{i} H_{1}(r, \theta)\right) \mathrm{e}^{\mathrm{i} k_{1} \Delta(\theta)+\mathrm{i} k_{2} \Psi(r, \theta)} .
$$

We retain $k_{3}$ in the governing equation so that we may discuss three-dimensional turbulence, however we impose $k_{3}=O\left(k_{1} M\right)$ and suppose $w^{2}>0$. The small $\theta$ scalings of the source terms $H_{0,1}$ are

$$
H_{0} \sim O\left(\frac{A_{1}}{a_{1} \theta^{2} r^{2}}\right), \quad H_{1} \sim O\left(\frac{A_{1} k_{1}}{a_{1}^{2} \theta^{2} r^{2}}\right),
$$

when constrained to the $\eta$-streamline, hence terms in (2.14) balance if $k=O\left(\tau^{-2}\right)$, and $\tilde{\phi}=O\left(k^{-1}\right)$. The constant $a_{1}$ is given in Appendix A.

We solve (2.14) using a Green's function satisfying

$$
\frac{\partial^{2} G}{\partial \theta^{2}}\left(\theta, \theta^{\prime}, r, r^{\prime}\right)+k^{2} \Omega(r)^{2} G\left(\theta, \theta^{\prime}, r, r^{\prime}\right)=\delta\left(\theta-\theta^{\prime}\right) \delta\left(r-r^{\prime}\right),
$$

where $\Omega(r)=w r$ and $\delta$ is the Dirac delta function. 
To determine appropriate boundary conditions for $(2.17)$, we consider the results from Durbin \& Hunt (1980, eq. 36) for turbulent interactions along the stagnation streamline, $\theta=0$. They found that the pressure decayed exponentially with $k$ on approach to the stagnation point. By asymptotically matching this exponentially small result with an inner limit of our small $\theta$ region, $\theta \rightarrow \theta^{c}$, we find we must impose the boundary conditions;

$$
\begin{aligned}
\tilde{\phi} & =0 \quad \text { at } & \theta & =\theta^{c}, \\
\frac{\partial \tilde{\phi}}{\partial \theta} & =0 \quad \text { at } & \theta & =\theta^{c} .
\end{aligned}
$$

For $\Omega^{2}>0$ the Green's function in (2.17) is oscillatory. If $\Omega^{2}<0$ we would obtain an exponentially decaying solution, which would be negligible, thus we only consider the case $\left|k_{3}\right| \leq M\left|k_{1}\right|$, which corresponds to sound waves with phase vector $\left(k_{1}, k_{2}, k_{3}\right)$ propagating to infinity rather than being evanescent. The Green's function is then

$$
G\left(\theta, \theta^{\prime}, r, r^{\prime}\right)=\left\{\begin{array}{cc}
0 & \text { if } \theta<\theta^{\prime} \\
\frac{1}{k \Omega(r)} \sin \left[\Omega(r) k\left(\theta-\theta^{\prime}\right)\right] \delta\left(r-r^{\prime}\right) & \text { if } \theta>\theta^{\prime}
\end{array} .\right.
$$

The solution of Eq. (2.14) comprises a particular solution, $\tilde{\phi}^{p}$, which takes care of the source term on the right hand side, and a homogeneous solution, $\tilde{\phi}^{h}$ that then ensures zero normal velocity on the rigid surface. Using our Green's function, (2.19), we find

$$
\tilde{\phi}^{p}=\frac{r}{k \sqrt{k_{1}^{\dagger 2} M^{2}-k_{3}^{\dagger 2}}} \int_{\theta}^{\epsilon}\left(H_{0}+\mathrm{i} H_{1}\right)\left(r, \theta^{\prime}\right) \mathrm{e}^{\mathrm{i} k\left(k_{1}^{\dagger} \Delta\left(\theta^{\prime}\right)+k_{3}^{\dagger} \Psi\left(r, \theta^{\prime}\right)\right)} \sin \left(k \Omega\left(\theta-\theta^{\prime}\right)\right) d \theta^{\prime},
$$

where $\epsilon$ is the the upper limit of validity of the small $\theta$ solution (defined by (A 19)). In the small $\theta$ approximation $\Psi=O(\eta)$, and hence we can neglect it compared to $\Delta$. Since $k$ is large we can apply the method of stationary phase to (2.20) (Bender \& Orszag 1978). The phase functions in $(2.20)$ are $g_{ \pm}\left(r, \theta^{\prime}\right)=k_{1}^{\dagger} \Delta\left(\theta^{\prime}\right) \pm \Omega(r)\left(\theta-\theta^{\prime}\right)$, which have points of stationary phase $\theta^{\prime}=\theta_{s}^{\prime}$ given by

$$
\theta_{s}^{\prime} \approx \frac{ \pm\left(1+b^{2}\right) k_{1}^{\dagger}}{\Omega a_{1}}
$$

We require $0<\theta<\theta_{s}^{\prime}<\epsilon$, hence on noting that $a_{1}<0$ (given in Appendix A) we see that only $g_{-}\left(r, \theta^{\prime}\right)$ yields a point of stationary phase within the allowed range, and hence

$$
\tilde{\phi}^{p} \sim \frac{-r}{2 \mathrm{i} k^{3 / 2} \sqrt{k_{1}^{\dagger 2} M^{2}-k_{3}^{\dagger 2}}} f_{0}\left(r, \theta_{s}^{0}\right) \sqrt{\frac{2 \pi}{g^{\prime \prime}\left(r, \theta_{s}^{0}\right)}} \mathrm{e}^{\mathrm{i} \pi / 4} e^{\mathrm{i} k \Omega\left(\theta_{s}^{0}-\theta\right)} .
$$

Evaluating $g_{+}$at an end point (which would yield the dominant contribution to its integral) would result in a term of $O\left(k^{-1 / 2}\right)$ smaller than the leading-order term given in $(2.22)$.

The homogeneous solution of (2.17) requires evaluation of the Green's function on the surface,

$$
\tilde{\phi}^{h}(r, \theta)=-\left.\int_{\eta}^{\epsilon} G\left(\theta^{\prime}, \theta, r^{\prime}, r\right) \frac{\partial \bar{\phi}^{h}}{\partial n}\right|_{r^{\prime}=\sqrt{\left(1+b^{2}\right)^{2} \cos ^{2} \theta^{\prime}+\left(1-b^{2}\right)^{2} \sin ^{2} \theta^{\prime}}} d \theta^{\prime} .
$$

The integration in (2.23) should be evaluated over the entire surface of the ellipse, however the greatest contribution to the integral arises from the region where $\theta$ is small since $\frac{\partial \tilde{\phi}^{p}}{\partial n}$ 
Interaction of turbulence with the leading-edge stagnation point of a thin aerofoil 7

is $O\left(k^{-1 / 2}\right)$ smaller when $\theta \gg \epsilon$ compared to when $\theta<\epsilon$. Also, $\boldsymbol{u}^{(I)} \cdot \boldsymbol{n}$ is largest in a region where the steady flow is slowed most significantly by the solid surface, which occurs in the region around the leading edge, therefore $\boldsymbol{u}^{(I)} \cdot \boldsymbol{n}$ is also larger for $\theta<\epsilon$ compared to when $\theta \gg \epsilon$. Evaluating the integral in (2.23) yields

$$
\tilde{\phi}^{h}(r, \theta)=\left.\frac{1}{k R\left(\theta^{h} ; r\right) w} \sin \left[k R\left(\theta^{h} ; r\right) w\left(\theta-\theta^{h}(r)\right)\right] \frac{\partial \tilde{\phi}^{h}}{\partial n}\right|_{\left(R\left(\theta^{h} ; r\right), \theta^{h}\right)},
$$

where

$$
\begin{aligned}
\theta^{h}(r) & =2 \arctan \left[\sqrt{\frac{1-6 b^{2}+b^{4}-r^{2}+4 b \sqrt{r^{2}-\left(1-b^{2}\right)^{2}}}{\left(1+b^{2}\right)^{2}-r^{2}}}\right], \\
R\left(\theta^{h} ; r\right) & =\sqrt{\left(1+b^{2}\right)^{2} \cos ^{2} \theta^{h}+\left(1-b^{2}\right)^{2} \sin ^{2} \theta^{h}}, \\
\frac{\partial \tilde{\phi}^{h}}{\partial n}(r, \theta) & =-\overline{\boldsymbol{u}}^{(I)} \cdot \boldsymbol{n}-\frac{\partial \tilde{\phi}^{p}}{\partial n} .
\end{aligned}
$$

The single high-frequency gust response, $\phi=\phi^{h}+\phi^{p}$ derived here is non-singular as the stagnation point is approached, $\theta, \eta \rightarrow 0, r \rightarrow 1+b^{2}$.

\subsubsection{Far Field Implications and Comparison with Previous Work}

We can match this inner solution to a large $r$ solution whilst remaining within the small $\theta$ region. We note from (2.21) that for large enough $r$ the point of stationary phase, $\theta_{s}=O\left(r^{-1}\right)$, falls outside the region of integration for the leading-order particular solution, hence the magnitude of the particular solution would drop by $O\left(k^{-1 / 2}\right)$. This is negligible to the orders retained in our calculations, so we are therefore left with $\phi=\phi^{h}$ as $r \rightarrow \infty$. We notice that $\theta^{h} \rightarrow \pi / 2$ for $r \rightarrow \infty$, and hence $\theta^{h}$ is also out of range of the limits of integration in (2.23), thus $\phi^{h} \rightarrow 0$ also. At first sight this prediction that the far-field sound directly upstream is negligible to the orders retained in our calculations seems incorrect, given that Tsai (1992) predicts the pressure to be $O\left(k^{-3 / 2}\right)$. However, we recall that our far-field solution is restricted to $\theta=O(\tau)$, which if incorporated into the result from Tsai (1992) (who has $\theta=O(1)$ ) also gives a reduction of the farfield magnitude by $O(\tau)$ (equivalent to $O\left(k^{-1 / 2}\right)$ ). It is also evident that this prediction of negligible pressure far upstream is consistent with Fig. 4.25 of Tsai (1992), which shows visually that directly upstream the far-field acoustic pressure is much smaller than elsewhere. We attribute this zero upstream far-field noise to distortion of the acoustic waves generated near the surface directly upstream; due to the steady flow any acoustic waves are diffracted away from the upstream direction by the time they approach the far field.

We can further extend our inner solution $\tilde{\phi}^{p}$ into the region outside of our predefined small $\theta$ region, and thereby asymptotically extend our solution into the leading-edge outer region as described by Tsai (1992). We once again use the method of stationary phase, but this time do not approximate the drift function, $\Delta$, since we have moved out of the region where (A 16) is valid. Further, $\Delta$ is now seen as a function of both $r$ and $\theta$ and we take $\theta=O(1)$ but $\theta<\pi / 2$. The stationary phase point, (2.21), now occurs at $k_{1}^{\dagger} \Delta^{\prime}\left(r, \theta_{s}\right)=\Omega \sim r$, hence $\Delta \sim r$ at the point of stationary phase. Thus

$$
\tilde{\phi}^{p} \sim \frac{r}{k} \frac{f\left(r, \theta_{s}\right)}{\sqrt{k \Delta^{\prime \prime}\left(r, \theta_{s}\right)}} \mathrm{e}^{\mathrm{i} k w r\left(\theta_{s}-\theta\right)}
$$


for large $k$. As we take $r \gg 1$, we see that $f\left(r, \theta_{s}\right) \sim \frac{\Delta}{r^{2}} \sim \frac{1}{r}$, which yields

$$
\phi^{p} \sim \frac{1}{k^{3 / 2} \sqrt{r}}
$$

for $\theta=O(1)$ and large $r$, which has the same order of magnitude in both $k$ and $r$ as the symmetric Joukowski aerofoil solutions in Tsai (1992) for high-frequency gusts. The contribution from $\phi^{h}$ is negligible, since when $r$ is large, $\theta^{h} \rightarrow \pi / 2$. We have therefore confirmed that our analytic solution in the small region close to the stagnation streamline is consistent with previous analytic solutions for high-frequency gust-aerofoil interactions.

\subsection{Low-Frequency Solution}

We now investigate the low-frequency limit, $k \ll 1$, of $(2.6 a)$. The wavelength of the gust is now much larger than the thickness of the ellipse, hence we expect that, to leading order, the solution resembles that of the flat plate, and is independent of the thickness of the ellipse. The governing equation for the low-frequency solution is given by (2.13) which we write as

$$
\frac{\partial^{2} \tilde{\phi}}{\partial \theta^{2}}=-r^{2} f(r, \theta)
$$

where $f(r, \theta)$ is still given by (2.15), and the scalings (2.16) still hold albeit $k$ is now small, so $H_{1} \ll H_{0}$. The conditions (2.18) still hold, and we also still require zero normal velocity on the surface of the ellipse. We assume $r$ is $O(1)$ in deducing (2.27), thus we have neglected terms of $O\left((k r)^{2}\right)$. We therefore cannot consider far-field low-frequency results $\left(r \gg k^{-1}\right)$, however due to the wavelength being large we would expect the farfield results to be a dipole that could be modelled by flat-plate interaction theory, which is well understood (Amiet 1975). Instead we are more interested in the low-frequency limit of the unsteady surface pressure spectrum.

The low-frequency particular solution to (2.27) satisfying (2.18) is

$$
\tilde{\phi}^{p}=r^{2} \int_{\theta^{c}}^{\theta} d \theta^{\prime} \int_{\theta^{c}}^{\theta^{\prime}} f\left(r, \theta^{\prime \prime}\right) d \theta^{\prime \prime} .
$$

The homogeneous solution, satisfying the zero normal velocity requirement on the boundary of the ellipse, is

$$
\tilde{\phi}^{h}=A(r)\left(\theta-\theta_{c}\right),
$$

where $A$ is an as yet undetermined function of $r$. On the surface for small $\theta$, to leading order,

and

$$
\boldsymbol{u}^{(I)} \cdot \boldsymbol{n}=\frac{A_{1} r^{c}}{r}=O(1)
$$

$$
\frac{\partial \tilde{\phi}^{h}}{\partial n} \approx \frac{1}{1-b^{2}}\left[A^{\prime}(r)\left(1-b^{2}\right)-\frac{2 \theta A(r)}{1+b^{2}}\right],
$$

hence on the surface of the ellipse we require $A(r)=O\left(\left(1-b^{2}\right) \theta^{-1}\right)$. It will be sufficient to know just the order of magnitude to deduce the effects of turbulence at leading order close to the stagnation point, therefore we do not explicitly solve for $\tilde{\phi}^{h}$.

\section{Effect of Turbulence and the Turbulent Spectra}

We now use our results for a single gust from the previous section to consider the effect of homogeneous weak turbulence from upstream interacting with the leading-edge 
Interaction of turbulence with the leading-edge stagnation point of a thin aerofoil 9 stagnation point of the ellipse (see Goldstein (1978) and Hunt (1973) for early work on this topic). We assume the three-dimensional upstream turbulent spectrum, $\Phi_{i, j}^{(\infty)}\left(k_{1}, k_{2}, k_{3}\right)$, is known. The one-dimensional turbulent velocity spectrum is defined by

$$
\Theta_{\nu, \mu}\left(\boldsymbol{x}, k_{1}\right)=\frac{1}{2 \pi} \int_{-\infty}^{\infty} R_{\nu, \mu}(\boldsymbol{x}, \tau) \mathrm{e}^{\mathrm{i} k_{1} \tau} d \tau,
$$

where $\nu$ and $\mu$ are $r, \theta$ or $z$.

$$
R_{\nu, \mu}(\boldsymbol{x}, \tau)=\left\langle u_{\nu}(\boldsymbol{x}, t) u_{\mu}(\boldsymbol{x}, t+\tau)\right\rangle
$$

is the one-point turbulent velocity correlation tensor, where the angle brackets denote time averaging. It relates to the known upstream turbulent spectrum via the equation

$$
\Theta_{\nu, \mu}\left(\boldsymbol{x}, k_{1}\right)=\int_{-\infty}^{\infty} \int_{-\infty}^{\infty} \overline{M_{\nu, j}} M_{\mu, n} \Phi_{j, n}^{(\infty)}(\boldsymbol{k}) d k_{2} d k_{3},
$$

where the over bar denotes complex conjugation, and the $M_{\alpha, i}$ are given in Goldstein (1978) via

$$
u_{\nu}=A_{j} M_{\nu, j}(r, \theta) \mathrm{e}^{\mathrm{i} k_{1}(\Delta-t)+\mathrm{i} k_{2} \Psi+\mathrm{i} k_{3} z} \quad \text { for } \quad \nu=r, \theta, z .
$$

We choose the von Kármán spectrum far upstream, as done by Goldstein (1978),

$$
\Phi_{j, n}^{(\infty)}=\alpha \frac{\left(k_{1}^{2}+k_{2}^{2}+k_{3}^{2}\right) \delta_{j n}-k_{j} k_{n}}{\left(g_{2} / l^{2}+k_{1}^{2}+k_{2}^{2}+k_{3}^{2}\right)^{17 / 6}}
$$

where

$$
\alpha=\frac{55 g_{1}\left\langle u_{\infty}^{2}\right\rangle}{36 \pi l^{2 / 3}}
$$

the $g_{i}$ are constants to be determined from experimental data, and $l$ denotes the integral lengthscale of the upstream turbulence. The magnitude of the unsteady flow far upstream is given by $u_{\infty}$.

We define the one-dimensional turbulent pressure spectrum analogously to the velocity spectrum as

$$
\Theta_{p p}\left(\boldsymbol{x}, k_{1}\right)=\int_{-\infty}^{\infty} \int_{-\infty}^{\infty} \overline{N_{i}} N_{j} \Phi_{i, j}^{(\infty)}(\boldsymbol{k}) d k_{2} d k_{3},
$$

where the $N_{i}$ are given via

$$
\frac{p}{\rho}=N_{j}(r, \theta) A_{j} \mathrm{e}^{\mathrm{i} k_{1}(\Delta-t)+\mathrm{i} k_{2} \Psi+\mathrm{i} k_{3} z},
$$

and the perturbation pressure can be found from the solutions for the unsteady velocity potential determined in Section 2 using

$$
p=-\rho_{0} \frac{D_{0} \phi}{D t} .
$$

We calculate $\Theta_{p p}$ in Appendix B.

\subsection{High-Frequency Pressure Spectrum in the Far Field}

In a similar manner to Section 2.2.1 we can extend our solution for $\Theta_{p p}$ into the far field for $\theta=O(1)$ (recall, in Section 2.2 we found that in the far field for $\theta=O(\tau)$ the solution was negligible). This yields

$$
\Theta_{p p} \sim \frac{2 g_{1}\left\langle u_{\infty}^{2}\right\rangle \sqrt{\pi}}{3 r} \frac{\Gamma\left(\frac{1}{3}\right)}{\Gamma\left(\frac{5}{6}\right)} \cos \theta \sin ^{4} \theta l^{2} M \begin{cases}g_{2}^{-4 / 3} & k_{1} l \ll 1 \\ \left(k_{1} l\right)^{-8 / 3} & k_{1} l \gg 1\end{cases}
$$


in the high and low reduced frequency limits.

These two expressions can be combined to give a composite function for $\Theta_{p p}$;

$$
\Theta_{p p} \sim \frac{2\left\langle u_{\infty}^{2}\right\rangle \sqrt{\pi} g_{1} l^{2} M \Gamma\left(\frac{1}{3}\right)}{3 r \Gamma\left(\frac{5}{6}\right)} \frac{g_{2}^{-4 / 3} \cos \theta \sin ^{4} \theta}{\left(1+\frac{\left(k_{1} l l^{8}\right.}{g_{2}^{4}}\right)^{1 / 3}}
$$

\subsection{High- and Low-Frequency Surface Pressure Spectrum}

The surface pressure spectrum can be found close to the leading-edge stagnation point when $\theta$ is small. We consider both the low- and high-frequency spectra.

\subsubsection{Low-Frequency}

The scattered pressure can be written as

$$
p=-\rho_{0} \mathrm{e}^{\mathrm{i} k_{1} t+\mathrm{i} k_{3} z}\left[-\mathrm{i} k_{1} \bar{\phi}+U_{r} \frac{\partial \tilde{\phi}}{\partial r}+\frac{U_{\theta}}{r} \frac{\partial \tilde{\phi}}{\partial \theta}\right]
$$

where $\tilde{\phi}=\tilde{\phi}^{h}+\tilde{\phi}^{p}$. Evaluating each term in (3.12) in the low-frequency regime and within the small $\theta$ region gives scalings

$$
p^{p}=O\left(1-b^{2}\right), \quad p^{h}=O(1),
$$

where $p^{p, h}$ are the contributions to the surface pressure due to the particular and homogeneous solutions respectively. Thus, in the thin ellipse limit, $b \rightarrow 1$, the homogeneous solution dominates the pressure on the surface of the ellipse near the nose. Hence, the interaction between the boundary and the gust generates the majority of the unsteady pressure. Since the solution does not depend on the thickness of the ellipse at leading order, we can assume that the wavelength of the gust is so large that it does not see an elliptical body in the steady flow, but rather a point source.

To compute $\Theta_{p p}$ we therefore follow Mish (2001), who considers spectra for a NACA 0015 aerofoil, based on the work for a flat plate done by Amiet (1975), and writes the pressure jump across a flat plate due to an incident gust as

$$
\Delta p^{*}(x, y, t)=2 \pi \rho_{0}^{*} U^{*} b^{*} w_{0}^{*} g\left(x, k_{1}, k_{3}\right) \mathrm{e}^{\mathrm{i} k_{3}^{*} z^{*}-k_{1}^{*} U^{*} t^{*}}
$$

in dimensionalised form. Here $g$ is the transfer function between the incident velocity and aerofoil pressure jump, and $w_{0}^{*}$ is magnitude of the incident gust velocity. The cross power spectral density at a given point is given by Mish (2001) as

$$
S_{q q}\left(x, x, 0, \omega^{*}\right)=4\left(2 \pi \rho_{0}^{*} b^{*}\right)^{2} \int_{-\infty}^{\infty} \int_{-\infty}^{\infty}\left|g\left(x,-\omega / U, k_{3}\right)\right|^{2} \Phi_{1,1}^{(\infty)}\left(-\omega^{*} / U^{*}, k_{2}^{*}, k_{3}^{*}\right) d k_{2}^{*} d k_{3}^{*},
$$

where $\omega^{*}$ is the circular frequency, $U^{*}$ is the steady free-stream velocity, and $b^{*}$ is the semi-chord length of the flat plate. Mish (2001) provides us with the transfer function for a thin aerofoil, which in our limit of small $k_{1}^{*}$ and small Mach number is

$$
g\left(x, k_{1}, k_{3}\right)=-\left(1-\sqrt{\frac{x}{2}}\left(1-\operatorname{erf}\left[\sqrt{2(2-x) k_{3}}\right]\right)\right) \frac{\mathrm{e}^{-\mathrm{i} k_{3} x}}{\pi^{3 / 2} \sqrt{x} \sqrt{k_{3}+\mathrm{i} k_{1}}} .
$$

Using this expression we can integrate (3.15) numerically. We are careful to choose parameters that are consistent with our scalings of thickness and proximity to the leading-edge stagnation point with respect to the frequency parameter $k$, and take values for $U, \omega$, $b$ and $\rho_{0}$ from Mish \& Devenport (2006) for comparison later in Section 4. We do not consider $k_{1} l \gg 1$ in the low-frequency case because it would not be realistic to allow a turbulent lengthscale to be so large, $l \gg k^{-1}$, for $k \ll 1$. 
SPL

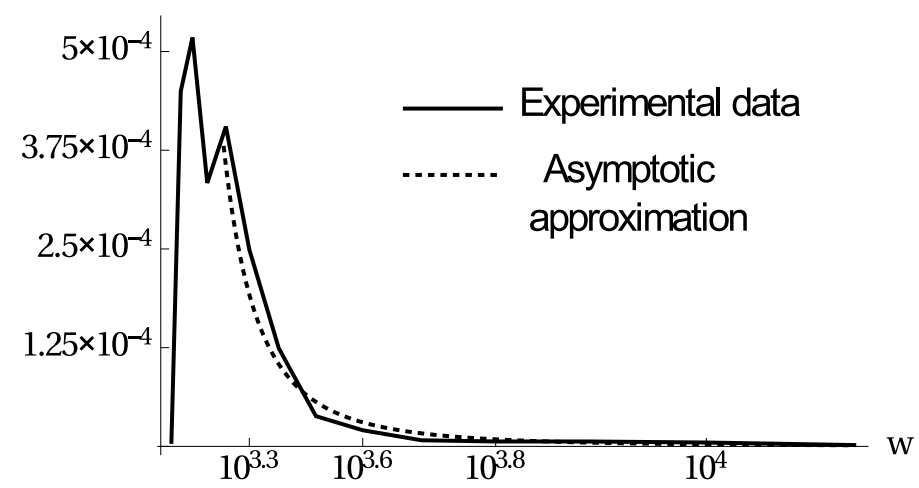

Figure 2: Sound pressure levels away from the leading edge of an SD7003 aerofoil measured experimentally, compared to the asymptotically obtained turbulent pressure spectrum. The horizontal axis measures (dimensional) frequency, $w=k_{1}^{*}$. The vertical axis measures the pressure level. Experimental data from (Geyer et al. 2012) comes courtesy of Thomas Geyer (pers. comm.).

\subsubsection{High-Frequency}

We now consider the behaviour of (B 4) for $k_{1} \gg 1$ to yield

$$
\Theta_{p p} \sim \frac{g_{1} \overline{u_{\infty}^{2}} \sqrt{\pi} \Gamma\left(\frac{1}{3}\right) l^{2} M}{3 \Gamma\left(\frac{5}{6}\right)\left(1-b^{2}\right)^{2}}\left\{\begin{array}{ll}
g_{2}^{-4 / 3} & k_{1} l \ll 1 \\
\left(k_{1} l\right)^{-8 / 3} & k_{1} l \gg 1
\end{array},\right.
$$

in the high-frequency limit. A composite function can be constructed similarly to the far-field high-frequency solution,

$$
\Theta_{p p} \sim \frac{g_{1} \overline{u_{\infty}^{2}} \sqrt{\pi} \Gamma\left(\frac{1}{3}\right) l^{2} M}{3 \Gamma\left(\frac{5}{6}\right)\left(1-b^{2}\right)^{2}} \frac{g_{2}^{-4 / 3}}{\left(1+\frac{\left(k_{1} l\right)^{8}}{g_{2}^{4}}\right)^{1 / 3}} .
$$

The above result now sheds light on an even smaller inner region to that considered by Tsai (1992), since here we are constrained to a narrow wedge around the stagnation point as opposed to allowing $O(1)$ values of $\theta$. This narrower region properly analyses the effects of the stagnation point on high-frequency incident turbulence, which were neglected in Tsai (1992).

\section{Comparison with experimental data}

Here we present comparisons of our asymptotic results, (3.11), (3.17) and (3.15) with experimental data.

In Figure 2 we compare our far-field high-frequency spectra, (3.11) to experimental sound pressure levels measured by Geyer et al. (2012). The parameters used are as follows; $U^{*}=35 \mathrm{~ms}^{-1}, g_{1}=0.2, g_{2}=0.56, M=0.09, \theta=\pi / 30, l=0.002$. These match the experimental setup, with $g_{1,2}$ chosen following Goldstein (1978). We see a good agreement for high frequencies, but at lower frequencies our asymptotic approximation no longer matches the experimental results. This is because in the experiment the upstream integral lengthscale of turbulence is fixed, while our high-frequency approximation has required us to vary $l$ so that $k_{1} l$ remains very small whilst $k_{1}$ remains very large. 


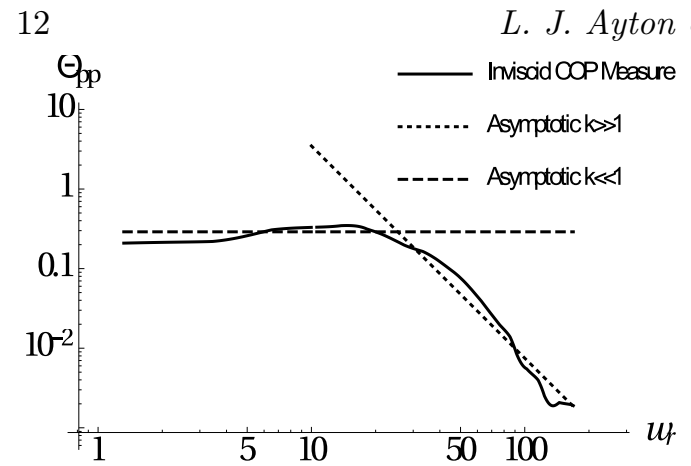

(a) Small grid, $l=0.0078 m$.

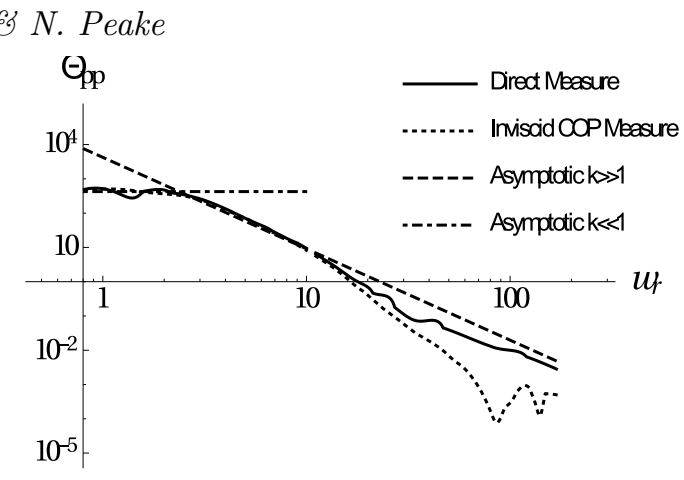

(b) Large grid, $l=0.0818 m$.

Figure 3: Asymptotically obtained pressure spectrum. Experimental data shows the surface pressure spectra measured on a NACA 0015 aerofoil. Coherent Output Power (COP) results give the inviscid spectra. Experimental results from (Mish \& Devenport 2006) are courtesy of William Devenport for two grid sizes yielding different integral lengthscales of turbulence.

On the surface of the ellipse, for small $\theta$, the low- and high-frequency limits of the turbulent pressure spectrum are given by (3.15) and (3.17) respectively. We compare these analytic solutions with the experimental data obtained by Mish \& Devenport (2006) in Figure 3. Mish \& Devenport (2006) introduced grid-generated turbulence upstream of a NACA 0015 aerofoil using a small or large grid to produce turbulence of different integral lengthscales, $l=0.0078$ (small grid) and $l=0.0818$ (large grid). We assume this turbulence is isotropic upstream, $U=30 \mathrm{~ms}^{-1}, b=0.305 \mathrm{~m}, M=0.09$, and $g_{1}=0.2, g_{2}=0.56$ again chosen following Goldstein (1978). The horizontal axis measures scaled frequency, $w_{r}=2 \pi k_{1} b U^{-1}$, and the vertical axis measures the (dimensional) surface pressure spectrum (normalised by $4 \pi U$ ). For the small grid, the turbulence is on the same lengthscale as the viscous boundary layer, therefore the directly measured (viscous) surface pressure is contaminated by boundary layer interactions, hence we only compare to the inviscid coherence output power (COP) measurements. The COP results, discussed in Mish (2001, pp. 75-77), extract the inviscid surface response from the measured pressure spectra, removing any turbulent boundary layer fluctuations. We see good agreement between the asymptotic results and the COP measurements in Figure 3a at both low and high frequencies. The large grid turbulence has a lengthscale much greater than that of the viscous boundary layer, hence for $w_{r} \lesssim 10$ we see good agreement between the directly measured, COP, and asymptotic results in Figure $3 \mathrm{~b}$. For $w_{r} \gtrsim 10$ the COP measurements diverge from the direct measurements indicating an onset of some viscous behaviour and hydrodynamic effects discussed in Mish \& Devenport (2006). The asymptotic result is still in reasonable agreement with direct measurements, but has some distinct differences indicating that alongside viscous interferences, we could also expect non-linear interactions to begin to take effect.

\section{Conclusions}

We have considered the effects of homogeneous isotropic turbulence from far upstream interacting with the leading-edge stagnation point of an aerofoil, by calculating analytic approximations for the turbulent pressure spectrum, both on the aerofoil surface close to the stagnation point, and in the far field close to the stagnation streamline. The analytic solution we have found requires the Mach number and body thickness to be small. This 
allows simplifications to be made, and leading-order approximations to be found. We identified the need to consider a new asymptotic region for the gust-aerofoil interaction problem because current analytic methods based on thin aerofoil theory predict a singularity at the leading-edge stagnation point which invalidates asymptotic assumptions. Our solution in a region close to the leading-edge stagnation point does not permit a singularity, and has been shown to be compatible with previous asymptotic solutions through a matching process in the high-frequency limit.

Both high- and low-frequency turbulent interactions were considered, and the resulting turbulent pressure spectra on the surface and in the far field showed good agreement with experimental data. For small scale turbulence, our analytic surface predictions agree well with inviscid measured data for all frequency ranges, whilst for large scale turbulence we have good agreement up to scaled frequency $w_{r} \approx 10$, after which non-linear effects may begin to be important. Below $w_{r} \approx 10$, both the directly measured and inviscid COP spectra of Mish \& Devenport (2006) agree since the viscous boundary layer is on a much smaller lengthscale than the upstream turbulence. We can conclude that the analytic model presented in this paper accurately predicts the inviscid response to both small and large scale upstream turbulence, provided viscous and non-linear effects are negligible. This approximation for the inviscid response can be used during post-processing of measured data to establish the effects of boundary layer interactions, without requiring additional data processing such as COP.

We have not provided results along the stagnation streamline itself, since these can be recovered from Durbin (1978), who found that along a stagnation streamline the turbulence pressure spectra decays exponentially due to piling up of eddies and strong cancellation of the corresponding pressure contributions. This has more recently been seen by Santana \& Schram (2015), where the high-frequency decay rate of the turbulence pressure spectrum increases with proximity to the stagnation point. Whilst the results from Durbin (1978) are specifically for bluff bodies with $O(1)$ thickness, the result along the stagnation streamline would also hold for thin bodies such as those discussed in this paper, since the stagnation streamline is unaffected by the conformal mapping from a circular cylinder to a thin elliptic cylinder.

\section{Acknowledgements}

The work in this paper was funded by EPSRC under grant EP/I010440/1. We are very grateful for this support.

\section{Appendix A. Stream Function and Drift Function}

Here we obtain the stream function, $\Psi$, and drift function, $\Delta$, for incompressible steady uniform flow around a thin elliptic cylinder, as required in Section 2 . We begin with steady flow around a circular cylinder of radius $R^{*}$ which far upstream is uniform with velocity $\boldsymbol{U}_{\infty}^{*}=U_{\infty}^{*} \hat{\boldsymbol{e}}_{x}$. This is mapped via the Joukowski transformation to give the corresponding flow around an elliptic cylinder. Velocities are non-dimensionalised with respect to $U_{\infty}^{*}$, and lengths with respect to $R^{*}$.

The (non-dimensional) velocity potential of the flow around the circular cylinder is given by

$$
\Phi(s, \varphi)=\left(s+\frac{1}{s}\right) \cos \varphi,
$$

where $(s, \varphi)$ are standard polar coordinates with origin at the centre of the cylinder. The 
velocity field is obtained from the potential, $\boldsymbol{U}_{\mathbf{0}}=\nabla \Phi$, and the streamfunction, $\Psi$, is then obtained as

$$
\Psi(s, \varphi)=\left(s-\frac{1}{s}\right) \sin \varphi .
$$

Since we wish to consider the flow around an elliptic cylinder, we apply the Joukowski transformation,

$$
z=\zeta+\frac{b^{2}}{\zeta}
$$

to map from a circular to an elliptical cylinder, where $\zeta=s \mathrm{e}^{\mathrm{i} \varphi}$, and $z=r \mathrm{e}^{\mathrm{i} \theta}$ define the $(r, \theta)$ polar coordinates for the elliptic geometry, centred on the centre of the ellipse, with $\theta=0$ corresponding to the upstream direction, and measured clockwise. The unit circular cylinder is mapped to an elliptic cylinder of minor axis $1-b^{2}$ and major axis $1+b^{2}$. The transformation of coordinates is given by

$$
\begin{aligned}
s & =\frac{1}{2 \cos \varphi}\left(-r \cos \theta-\sqrt{r^{2} \cos ^{2} \theta-4 b^{2} \cos ^{2} \varphi}\right), \\
\sin ^{2} \varphi & =\frac{4 b^{2}-r^{2}}{8 b^{2}}+\frac{1}{2} \sqrt{\frac{\left(r^{2}-4 b^{2}\right)^{2}}{16 b^{4}}+\frac{r^{2} \sin ^{2} \theta}{b^{2}}},
\end{aligned}
$$

and close to the leading-edge stagnation streamline, $\theta \approx 0$, this is approximated by

$$
\begin{array}{r}
s \approx \frac{1}{2}\left(\sqrt{r^{2}-4 b^{2}}+r\right), \\
\sin \varphi \approx \frac{r \sin \theta}{\sqrt{r^{2}-4 b^{2}}} .
\end{array}
$$

We obtain the streamfunction for the ellipse by applying the transform (A $3 d$ ) to (A 2).

The drift function is defined by (2.4). In order to evaluate $\Delta$ we march along a streamline by integrating the relation

$$
d t=\frac{r d \theta}{U_{\theta}},
$$

as done in Lighthill (1956). To do this we write $r$ in terms of $\theta$ along a given streamline close to the leading-edge stagnation streamline, $\Psi=0$. We choose

$$
\eta=\Psi(r, \theta)
$$

for $\eta \ll 1$ and solve (A 5 ) for $r$ by expanding

$$
r=r_{0}+\eta r_{1}+O\left(\eta^{2}\right)
$$

in a similar way to Lighthill (1956). Equating (A 5) at each order using (A 6) allows us to solve for $r_{0,1}$;

$$
\begin{aligned}
& r_{0}=1+b^{2}, \\
& r_{1}=\frac{\left(1-b^{2}\right)^{2}}{2\left(1+b^{2}\right)} \csc \theta .
\end{aligned}
$$

We notice from (A 7 ) that (A 6 ) is in fact a series in $\eta / \sin \theta$ so it is only valid if $1 \gg \theta>\eta$, hence our solution is bounded away from the stagnation streamline. To bound $\theta$ values away from the stagnation point, we introduce $\theta^{c}$ as the lower limit of allowable small $\theta$ values. As illustrated in Figure 1 , the $\Psi=\eta$ streamline in the region $\theta \leq \theta^{c}$ is approximately a straight line from upstream infinity, which arises from the uniform flow. 
Interaction of turbulence with the leading-edge stagnation point of a thin aerofoil 15

Hence we can treat this region as Goldstein (1978) does, so when calculating the drift we use a uniform flow approximation for $\theta \leq \theta^{c}$ and our expansion (A 6) for $\theta>\theta^{c}$. Specifically, the point on the $\eta$ streamline, $\left(r^{c}, \theta^{c}\right)$, is approximated by

$$
\begin{aligned}
\theta^{c}= & \frac{\eta}{r^{c}}, \\
r^{c}= & \frac{4\left(1+b^{2}\right)^{4}}{1+6 b^{2}+8 b^{4}+2 b^{6}-b^{8}+\left(1+b^{2}\right)^{2} \sqrt{50 b^{4}-24 b^{6}-b^{8}-8 b^{2}-1}}+O\left(\eta^{2}\right), \\
& \sim 2-2(1-b) .
\end{aligned}
$$

We later neglect terms of $O(\eta)$ therefore our solution will be independent of $\theta^{c}$, however we define it here to specifically bound our region of interest away from the $\Psi=0$ streamline.

We note that Durbin (1978) considered turbulent interactions with a bluff body along $\Psi=0$ and found that close to the leading-edge stagnation point of the body, the turbulent pressure spectrum decays exponentially with frequency. Since we anticipate an identical result along $\Psi=0$, we only consider $\Psi=\eta \neq 0$.

Using (A 6) we can write the velocity component $u_{\theta}$ as

$$
u_{\theta}=a_{1} \sin \theta+\eta\left(a_{2}+a_{3} f(\theta)+a_{4} \cos 2 \theta\right) \frac{1}{f(\theta)},
$$

where

$$
f(\theta)=\sqrt{\left(1-b^{2}\right)^{2}-\left(1+b^{2}\right)^{2} \sin ^{2} \theta} .
$$

We require $\theta<2 \arctan \left(\frac{1-b}{1+b}\right)$ to ensure that $f$ is real.

The $a_{i}$ are constants given by

$$
\begin{aligned}
& a_{1}=-\frac{2\left(1+b^{2}\right)}{\left(1-b^{2}\right)^{2}} \\
& a_{2}=-\frac{2\left(b^{2}-6 b^{4}+b^{6}\right)}{\left(1+b^{2}\right)\left(1-b^{2}\right)^{3}} \\
& a_{3}=\frac{\left(b^{4}+6 b^{2}+1\right)}{\left(1+b^{2}\right)\left(1-b^{2}\right)^{2}} \\
& a_{4}=-\frac{2 b^{2}\left(1+b^{2}\right)}{\left(1-b^{2}\right)^{2}}
\end{aligned}
$$

Using (A 6) we obtain the asymptotic expression for $u_{\theta}$ for $r$ close to the surface, in the form

$$
\frac{r}{u_{\theta}} \sim \frac{\left(1+b^{2}\right)}{a_{1}} \csc \theta+\eta \csc ^{2} \theta \frac{1}{a_{1}^{2}}\left[\frac{a_{1}\left(1-b^{2}\right)^{2}}{2\left(1+b^{2}\right)}-\frac{1+b^{2}}{f(\theta)}\left(a_{2}+a_{4} \cos 2 \theta+a_{3} f(\theta)\right)\right] .
$$

Finally, we insert (A 15) into (A 4) and integrate to obtain the drift function $\Delta(\theta) \approx$ const. $-r \cos \theta+\frac{\left(1+b^{2}\right)}{a_{1}} I_{1}+\frac{\eta}{a_{1}^{2}}\left(a_{1} \frac{\left(1-b^{2}\right)^{2}}{\left(1+b^{2}\right)} I_{2}-\left(1+b^{2}\right)\left[a_{2} I_{4}+a_{4} I_{3}+a_{3} I_{2}\right]\right)$, 
where

$$
\begin{aligned}
I_{1}= & \int \csc \theta d \theta=\log [\tan (\theta / 2)], \\
I_{2}= & \int \csc ^{2} \theta d \theta=-\cot \theta, \\
I_{3}= & \int \frac{\csc ^{2} \theta}{f(\theta)} \cos 2 \theta d \theta= \\
& \frac{g(\theta) \cot \theta}{\sqrt{2}\left(1-b^{2}\right)^{2}}-\frac{1}{\left(1-b^{2}\right)}\left[F\left(\theta \mid \frac{\left(b^{2}+1\right)^{2}}{\left(1-b^{2}\right)^{2}}\right)+E\left(\theta \mid \frac{\left(b^{2}+1\right)^{2}}{\left(1-b^{2}\right)^{2}}\right)\right], \\
I_{4}= & \int \frac{\csc ^{2} \theta}{f(\theta)} d \theta=\frac{1}{\sqrt{2}\left(1-b^{2}\right)^{2} g(\theta)}\left\{\left(b^{2}+1\right)^{2} \sin 2 \theta-2\left(1-b^{2}\right)^{2} \cot \theta\right. \\
& \left.+\sqrt{2}\left(1-b^{2}\right) g(\theta) F\left(\theta \mid \frac{\left(b^{2}+1\right)^{2}}{\left(1-b^{2}\right)^{2}}\right)-\sqrt{2}\left(1-b^{2}\right) g(\theta) E\left(\theta \mid \frac{\left(b^{2}+1\right)^{2}}{\left(1-b^{2}\right)^{2}}\right)\right\},
\end{aligned}
$$

with

$$
g(\theta)=\sqrt{b^{4}+\left(b^{2}+1\right)^{2} \cos 2 \theta-6 b^{2}+1} .
$$

The quantity within the square root defined in $g(\theta)$ is positive subject to the same constraint as before,

$$
\theta<2 \arctan \left(\frac{1-b}{1+b}\right) \equiv \epsilon .
$$

We use (A 19) to define $\epsilon$, the upper limit of $\theta$. In (A 17), $F(x \mid m)$ and $E(x \mid m)$ denote elliptic integrals of the first and second kind respectively (Abramowitz \& Stegun 1964, pp. 589). The constant in (A 16) depends on $\eta, r^{c}$ and $\theta^{c}$. For our calculations we only require derivatives of $\Delta$ therefore we do not explicitly calculate the constant.

The integrals, (A 17), should be expanded for small $\theta$ to obtain an expression for $\Delta$ near the leading-edge stagnation point. These expansions are

$$
\begin{aligned}
& I_{1} \sim \log \left[\frac{\theta}{2}\right]+\frac{\theta^{2}}{12}+O\left(\theta^{3}\right), \\
& I_{2} \sim-\frac{1}{\theta}+\frac{\theta}{3}+O\left(\theta^{3}\right), \\
& I_{3} \sim-\frac{1}{\left(1-b^{2}\right) \theta}-\frac{\left(7 b^{4}-26 b^{2}+7\right) \theta}{6\left(1-b^{2}\right)^{3}}+O\left(\theta^{3}\right), \\
& I_{4} \sim-\frac{1}{\left(1-b^{2}\right) \theta}+\frac{\left(5 b^{4}+2 b^{2}+5\right) \theta}{6\left(1-b^{2}\right)^{3}}+O\left(\theta^{3}\right) .
\end{aligned}
$$

\section{Appendix B. Turbulent Pressure Spectrum}

The turbulent pressure spectrum is given by (3.7). We write the $N_{i}$ (defined in (3.8)) as

$$
N_{1}=\left(n_{0}+n_{1} \sqrt{k_{1}^{2} M^{2}-k_{3}^{2}}\right) \mathrm{e}^{-\mathrm{i} r \sqrt{k_{1}^{2} M^{2}-k_{3}^{2}}\left(\theta-\theta_{s}^{0}\right)}+n_{2} \cos \left[R \sqrt{k_{1}^{2} M^{2}-k_{3}^{2}}\left(\theta-\theta^{h}\right)\right]
$$


Interaction of turbulence with the leading-edge stagnation point of a thin aerofoil 17

$$
\begin{gathered}
+\frac{n_{3}}{\sqrt{k_{1}^{2} M^{2}-k_{3}^{2}}} \sin \left[R \sqrt{k_{1}^{2} M^{2}-k_{3}^{2}}\left(\theta-\theta^{h}\right)\right], \\
N_{2}=n_{4} \cos \left[R \sqrt{k_{1}^{2} M^{2}-k_{3}^{2}}\left(\theta-\theta^{h}\right)\right]+\frac{n_{5}}{\sqrt{k_{1}^{2} M^{2}-k_{3}^{2}}} \sin \left[R \sqrt{k_{1}^{2} M^{2}-k_{3}^{2}}\left(\theta-\theta^{h}\right)\right],
\end{gathered}
$$

where

$$
\begin{aligned}
n_{0} & =\frac{\mathrm{i} k_{1} C}{k^{3 / 2}}-\frac{U_{0, r}}{k^{3 / 2}}\left[\frac{\partial C}{\partial r}+\mathrm{i} k_{1} C \frac{\partial \Delta\left(\theta_{s}^{0}\right)}{\partial r}\right], & n_{1} & =\frac{\mathrm{i} C \theta}{k^{3 / 2}} U_{0, r}+\frac{\mathrm{i} C}{k^{3 / 2}} U_{0, \theta}, \\
n_{2} & =U_{0, r} \frac{F_{1}}{R} \frac{\partial}{\partial r}\left[R\left(\theta^{h}-\theta_{s}^{0}\right)\right]+\frac{F_{1}}{r} U_{0, \theta}, & n_{3} & =\mathrm{i} U_{0, r} \frac{F_{1}}{R} k_{1} \frac{\partial \Delta\left(R, \theta^{h}\right)}{\partial r}, \\
n_{4} & =U_{0, r} \frac{F_{2}}{R} \frac{\partial}{\partial r}\left[R\left(\theta^{h}-\theta_{s}^{0}\right)\right]+\frac{F_{2}}{r} U_{0, \theta}, & n_{5} & =\mathrm{i} U_{0, r} \frac{F_{2}}{R} k_{1} \frac{\partial \Delta\left(R, \theta^{h}\right)}{\partial r},
\end{aligned}
$$

and

$$
\begin{aligned}
\left.\boldsymbol{u}^{(I)} \cdot \boldsymbol{n}\right|_{\left(R, \theta^{h} ; r\right)} & =A_{i} F_{i}\left(R, \theta^{h} ; r\right) \mathrm{e}^{\mathrm{i} k_{1} \Delta+\mathrm{i} k_{2} \Psi+\mathrm{i} k_{3} z}, \\
\tilde{\phi}^{p} & =\frac{A_{1} C\left(r, t, k_{1}\right)}{k^{3 / 2}} \mathrm{e}^{\mathrm{i} k_{1} \Delta\left(r, \theta_{s}^{0}\right)+\mathrm{i} k_{2} \Psi\left(r, \theta_{s}^{0}\right)-\mathrm{i} r \sqrt{k_{1}^{2} M^{2}-k_{3}^{2}}\left(\theta-\theta_{s}^{0}\right)}, \\
\boldsymbol{U}_{\mathbf{0}} & =\left(U_{0, r}, U_{0, \theta}, U_{0, z}\right) .
\end{aligned}
$$

Therefore integrating (3.7) gives

$$
\begin{aligned}
\Theta_{p p} \approx & \frac{12 \alpha k_{1} M \sqrt{\pi} \Gamma\left(\frac{1}{3}\right)}{55 \Gamma\left(\frac{5}{6}\right)}\left(\frac{l^{2}}{g_{2}+k_{1}^{2} l^{2}}\right)^{4 / 3}\left[2-{ }_{2} F_{1}\left(\frac{1}{3}, \frac{1}{2}, \frac{3}{2} ;-\frac{k_{1}^{2} l^{2} M^{2}}{g_{2}-k_{1}^{2} l^{2}}\right)\right]\left|n_{0}\right|^{2} \\
& +\frac{72 \alpha \sqrt{\pi} \Gamma\left(\frac{1}{3}\right)}{55 \Gamma\left(\frac{5}{6}\right)}\left(\frac{l^{2}}{g_{2}+k_{1}^{2} l^{2}}\right)^{1 / 3}\left[1-{ }_{2} F_{1}\left(\frac{1}{3}, \frac{1}{2}, \frac{3}{2} ;-\frac{k_{1}^{2} l^{2} M^{2}}{g_{2}-k_{1}^{2} l^{2}}\right)\right]\left|n_{1}\right|^{2} \\
& +\frac{36 \alpha \pi^{3 / 2} \Gamma\left(\frac{1}{3}\right)}{55 \Gamma\left(\frac{5}{6}\right)}\left(\frac{l^{2}}{g_{2}+k_{1}^{2} l^{2}}\right)^{1 / 3}\left[{ }_{2} F_{1}\left(\frac{-2}{3}, \frac{1}{2}, 1 ;-\frac{k_{1}^{2} l^{2} M^{2}}{g_{2}-k_{1}^{2} l^{2}}\right)\right. \\
& \left.+\frac{4 k_{1} \alpha \sqrt{\pi} \Gamma\left(\frac{1}{3}\right)}{55 l^{2} \Gamma\left(\frac{5}{6}\right)}\left[8 k_{1}^{2} l^{2}\left|n_{4}\right|^{2} J_{1}+n_{2}^{2} J_{2}+\frac{1}{3}, \frac{3}{2} ;-\frac{k_{1}^{2} l^{2} M^{2}}{g_{2}-k_{1}^{2} l^{2}}\right)\right] \operatorname{Re}\left(n_{0} n_{1}^{*}\right) \\
& \left.\left.+\frac{2\left|n_{3}\right|}{k_{1}}\left(\operatorname{Im}\left(n_{1} J_{3}^{*}\right)+\operatorname{Re}\left(n_{0} J_{5}^{*}\right)\right)+\operatorname{Im}\left(n_{0} J_{7}\right)\right)+\frac{8 l^{2}}{k_{1}^{2}}\left|n_{5}\right|^{2} J_{6}+\frac{\left|n_{3}\right|^{2}}{k_{1}^{2}} J_{8}\right],
\end{aligned}
$$

where the function ${ }_{2} F_{1}$ denotes a hypergeometric function (Abramowitz \& Stegun 1964, pp. 556), and $\boldsymbol{U}_{\mathbf{0}}$ is the velocity field due to uniform flow around an ellipse as calculated in Appendix A. When integrating over $k_{3}$ to obtain the pressure spectra, we take only $\left|k_{3}\right| \leq M\left|k_{1}\right|$ to ensure an oscillatory solution for $\tilde{\phi}$ rather than an exponentially decaying one.

The $J_{i}$ in (B 4) are defined by

$$
J_{1}=\int_{0}^{M}\left(1+s^{2}\right)\left(\frac{l^{2}}{g_{2}+k_{1}^{2}\left(1+s^{2}\right) l^{2}}\right)^{7 / 3} \cos ^{2}\left[R k_{1} \sqrt{M^{2}-s^{2}}\left(\theta-\theta^{h}\right)\right] d s
$$


18 L. J. Ayton \& N. Peake

$J_{2}=\int_{0}^{M}\left(\frac{l^{2}}{g_{2}+k_{1}^{2}\left(1+s^{2}\right) l^{2}}\right)^{7 / 3}\left(3 g_{2}+k_{1}^{2}\left(3+11 s^{2}\right) l^{2}\right) \cos ^{2}\left[R k_{1} \sqrt{M^{2}-s^{2}}\left(\theta-\theta^{h}\right)\right] d s$,

$$
\begin{gathered}
J_{3}=\int_{0}^{M} \quad \sqrt{M^{2}-s^{2}}\left(\frac{l^{2}}{g_{2}+k_{1}^{2}\left(1+s^{2}\right) l^{2}}\right)^{7 / 3}\left(3 g_{2}+k_{1}^{2}\left(3+11 s^{2}\right) l^{2}\right) \\
\cos \left[R k_{1} \sqrt{M^{2}-s^{2}}\left(\theta-\theta^{h}\right)\right] \mathrm{e}^{\mathrm{i} r k_{1} \sqrt{M^{2}-s^{2}}\left(\theta-\theta_{s}^{0}\right)} d s,
\end{gathered}
$$

$J_{4}=\int_{0}^{M}\left(\frac{l^{2}}{g_{2}+k_{1}^{2}\left(1+s^{2}\right) l^{2}}\right)^{7 / 3}\left(3 g_{2}+k_{1}^{2}\left(3+11 s^{2}\right) l^{2}\right) \mathrm{e}^{\mathrm{i} r k_{1} \sqrt{M^{2}-s^{2}}\left(\theta-\theta_{s}^{0}\right)}$

$$
\cos \left[R k_{1} \sqrt{M^{2}-s^{2}}\left(\theta-\theta^{h}\right)\right] d s,
$$

$J_{5}=\int_{0}^{M} \frac{1}{\sqrt{M^{2}-s^{2}}}\left(\frac{l^{2}}{g_{2}+k_{1}^{2}\left(1+s^{2}\right) l^{2}}\right)^{7 / 3}\left(3 g_{2}+k_{1}^{2}\left(3+11 s^{2}\right) l^{2}\right)$

$$
\sin \left[R k_{1} \sqrt{M^{2}-s^{2}}\left(\theta-\theta^{h}\right)\right] d s,
$$

$J_{6}=\int_{0}^{M} \frac{1+s^{2}}{M^{2}-s^{2}}\left(\frac{l^{2}}{g_{2}+k_{1}^{2}\left(1+s^{2}\right) l^{2}}\right)^{7 / 3} \sin ^{2}\left[R k_{1} \sqrt{M^{2}-s^{2}}\left(\theta-\theta^{h}\right)\right] d s$,

$J_{7}=\int_{0}^{M} \frac{1}{\sqrt{M^{2}-s^{2}}}\left(\frac{l^{2}}{g_{2}+k_{1}^{2}\left(1+s^{2}\right) l^{2}}\right)^{7 / 3}\left(3 g_{2}+k_{1}^{2} l^{2}\left(3+11 s^{2}\right)\right) \mathrm{e}^{\mathrm{i} r k_{1} \sqrt{M^{2}-s^{2}}\left(\theta-\theta_{s}^{0}\right)}$ $\sin \left[R k_{1} \sqrt{M^{2}-s^{2}}\left(\theta-\theta^{h}\right)\right] d s$,

$J_{8}=\int_{0}^{M} \frac{1}{M^{2}-s^{2}}\left(\frac{l^{2}}{g_{2}+k_{1}^{2}\left(1+s^{2}\right) l^{2}}\right)^{7 / 3}\left(3 g_{2}+k_{1}^{2} l^{2}\left(3+11 s^{2}\right)\right)$

$$
\sin ^{2}\left[R k_{1} \sqrt{M^{2}-s^{2}}\left(\theta-\theta^{h}\right)\right] d s \text {. }
$$

In the far-field, $\Theta_{p p}$ is dominated by the vortical source, i.e. terms arising from $\tilde{\phi}^{p}$, hence only $n_{0,1}$ terms contribute. These are given by

$$
\begin{aligned}
& n_{0} \sim \frac{\sqrt{2 \pi} \mathrm{e}^{-\mathrm{i} \pi / 4} \mathrm{i} k_{1}}{\sqrt{M^{2} k_{1}^{2}-k_{3}^{2}} \sqrt{k r}} \sqrt{\cos \theta} \sin ^{2} \theta \\
& n_{1} \sim \mathrm{i} \sqrt{2 \pi} \sqrt{\frac{\cos \theta}{k r}} \frac{\mathrm{e}^{-\mathrm{i} \pi / 4}}{\sqrt{M^{2} k_{1}^{2}-k_{3}^{2}}}(\theta \cos \theta-\sin \theta)
\end{aligned}
$$

On the surface, dominant terms arise due to horizontal blocking, hence $n_{4,5} \sim 0$. Remaining terms are

$$
\begin{aligned}
& n_{0} \sim \frac{-\sqrt{\pi} \mathrm{ie}^{-\mathrm{i} \pi / 4}}{\sqrt{k_{1}}\left(1-b^{2}\right)}, \\
& n_{1} \sim \frac{2 \mathrm{i} \theta \sqrt{\pi} \mathrm{e}^{-\mathrm{i} \pi / 4}}{k_{1}^{3 / 2}\left(1-b^{2}\right)}, \\
& n_{2} \sim \frac{4 \theta}{1-b^{4}}, \\
& n_{3} \sim 0 .
\end{aligned}
$$


Interaction of turbulence with the leading-edge stagnation point of a thin aerofoil 19

\section{REFERENCES}

Abramowitz, M. \& Stegun, I. A. 1964 Handbook of Mathematical Functions: With Formulas, Graphs, and Mathematical Tables. Courier Dover Publications.

Allampalli, V., Hixon, R., Nallasamy, M. \& Sawyer, S. D. 2009 High-accuracy largestep explicit Runge-Kutta (HALE-RK) schemes for computational aeroacoustics. Journal of Computational Physics 228, 3837-3850.

Amiet, R.K. 1974 Compressibility effects in unsteady thin-airfoil theory. AIAA Journal 12, $252-255$.

Amiet, R.K. 1975 Acoustic radiation from an airfoil in a turbulent stream. Journal of Sound and Vibration 41, 407-420.

Bender, C. M. \& Orszag, S. A. 1978 Advanced Mathematical Methods for Scientists and Engineers. Springer.

Darwin, C. G. 1953 Note on hydrodynamics. Proc. Camb. Phil. Soc. 49, 342-354.

Durbin, P. A. \& Hunt, J. C. R. 1980 On surface pressure fluctuations beneath turbulent flow round bluff bodies. Journal of Fluid Mechanics 100, 161-184.

Durbin, P. A. 1978 Rapid Distortion Theory of Turbulent Flows. PhD thesis, University of Cambridge.

Durbin, P. A. \& Pettersson Reif, B. A. 2001 Statistical Theory and Modeling for Turbulent Flows. Wiley.

Geyer, T., Sarradj, E. \& Giesler, J. 2012 Application of a beamforming technique to the measurement of airfoil leading edge noise. Advances in Acoustics and Vibration 2012, 1-16.

Goldstein, M. E. 1978 Unsteady vortical and entropic distortions of potential flows round arbitrary obstacles. Journal of Fluid Mechanics 89, 433-468.

Goldstein, M. E. \& Atassi, H. 1976 A complete second-order theory for the unsteady flow about an airfoil due to a periodic gust. Journal of Fluid Mechanics 74, 741-765.

Hixon, R., Golubev, V., Mankbadi, R. R., Scott, J. R., Sawyer, S. \& Nallasamy, M. 2006 Application of a nonlinear computational aeroacoustics code to the gust-airfoil problem. AIAA Journal 44, 323-328.

Hunt, J. C. R. 1973 A theory of turbulent flow round two-dimensional bluff bodies. Journal of Fluid Mechanics 61, 625-706.

Kolmogorov, A. N. 1941 The local structure of turbulence in incompressible viscous fluid for very large Reynolds numbers. Doklady Akademiia Nauk SSSR 30, 301-305.

Lighthill, M J 1956 Drift. Journal of Fluid Mechanics 1, 31-53.

Mish, P. F. 2001 Mean Loading and Turbulence Scale Effects on the Surface Pressure Fluctuations Occurring on a NACA 0015 Airfoil Immersed in grid Generated Turbulence. PhD thesis, Virginia Polytechnic Institute and State University.

Mish, P. F. \& Devenport, W. J. 2006 An experimental investigation of unsteady surface pressure on an airfoil in turbulence - Part 1: effects of mean loading. Journal of Sound and Vibration. 296, 417-446.

Myers, M. R. 1987 Effect of airfoil mean loading on high-frequency gust interaction noise. $\mathrm{PhD}$ thesis, University of Arizona.

Myers, M. R. \& Kerschen, E. J. 1997 Influence of camber on sound generation by airfoils interacting with high-frequency gusts. Journal of Fluid Mechanics 353, 221-259.

Santana, L. D. \& Schram, C. 2015 Airfoil noise prediction from 2D3C PIV data. 21st AIAA/CEAS Aeroacoustics Conference, Dallas, TX AIAA 2015-2203.

SEARS, W. R. 1941 Some aspects of non-stationary airfoil theory and its practical applications. Journal of the Aeronautical Sciences 8, 104-188.

Tsai, C-T. 1992 Effect of Airfoil Thickness on High-Frequency Gust Interaction Noise. PhD thesis, University of Arizona.

Van Dyke, M. 1975 Perturbation methods in fluid mechanics. Parabolic Press. 